\title{
Use of Mouthguards by Amateur Basketball Athletes in Greece and The USA
}

\author{
Elena-Lito Exarchou ${ }^{1^{*}}$, Ioannis Kotsanos ${ }^{2}$ and Nikolaos Kotsanos ${ }^{1}$ \\ ${ }^{1}$ Department of Pediatric Dentistry, School of Dentistry, Aristotle University of Thessaloniki, Greece \\ ${ }^{2}$ Fitness and Wellness Program, Park University, USA
}

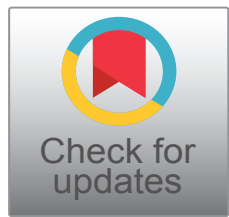

*Corresponding author: Elena-Lito Exarchou, Department of Pediatric Dentistry, School of Dentistry, Aristotle University of Thessaloniki, Sp.Spyridi 2-6, 38221, Volos, Greece, Tel: (+30)-2421181088

\begin{abstract}
Background: Mouthguard use reduces the consequences of oral trauma in sports with intense physical contact. The use of mouthguards is recommended by the Academy for Sports Dentistry for all sports involving physical contact and collision, and by the American Academy of Pediatric Dentistry for sports in which orofacial injury can occur, e.g. basketball. The aim of this study is to evaluate mouthguard use and relative knowledge by male amateur Greek and collegiate American basketball athletes.
\end{abstract}

Methods: In total 215 questionnaires were completed by almost equal numbers of male athletes from mainland Greece and central US colleges (mean age 20.5 and 20.8 respectively). They included closed and open-ended questions on the awareness, usage, acceptance and effectiveness of mouthguards. Answers were listed and analyzed with descriptive statistics and chi-square tests.

Results: The use of protective mouthguards in basketball was low $(12 \%$ in both samples) despite high percentages of informed athletes (54.8\% Greek, 86.5\% American). The dentist played a significantly $(p<0.01)$ more active role in promoting mouthguards in the USA (43.5\%) than in Greece $(18.8 \%)$. The most commonly reported anatomical locations of injury were the same in both countries: Lip injuries followed by trauma to the teeth and tongue. All athletes wearing a mouthguard felt protected from more serious injury.

Conclusions: Mouthguard use by Greek and USA samples of young amateur basketball athletes was low. There is a strong argument for improving awareness and encouraging mouthguard use promotion by dentists, especially in Greece.

\section{Keywords}

Mouthguard, Basketball, Injuries

\section{Introduction}

Over $30 \%$ of all dental trauma can be attributed to sports injuries. Although dental-related injuries occur less frequently than other sport injuries such as sprains and strains, treatment is invariably costly [1]. Moreover, players with dental injuries can suffer more permanent damage, which can affect both their ability to play and their well-being [2]. For these reasons it is important that dental-related sports injuries be addressed.

It is acknowledged that basketball, which is played by $11 \%$ of the world's population, is a sport that involves direct and indirect physical contact between players as well as between ball and player, with a high rate of orofacial injuries as a result [3]. Many studies maintain that basketball players suffer the highest degree of dental injury [4,5]. Given the potential risk of orofacial trauma, the Academy of Sports Dentistry and the American Academy of Pediatric Dentistry both advocate mouthguard use for basketball players in their guidelines [6]. When used, mouthguards safeguard the teeth and soft tissue by: a) Preventing laceration of tongue, lips and cheeks, b) Lowering the risk of injury to both anterior and posterior teeth and c) Reducing the likelihood of concussion resulting from impact to the jaw [7]. A decline in the incidence of dental injuries has been documented in sports activities such as American football and ice hockey, where mouthguard use is compulsory [8]. It seems to follow therefore that the rate of dental injury in basketball could be reduced by promoting mouthguard use for all basketball players [9].

It is noteworthy that in spite of their ease of

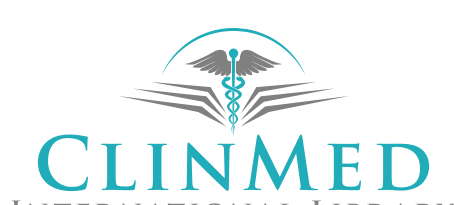

INTERNATIONAL LIBRARY
Citation: Exarchou EL, Kotsanos I, Kotsanos N (2019) Use of Mouthguards by Amateur Basketball Athletes in Greece and The USA. Trauma Cases Rev 5:071. doi.org/10.23937/2469-5777/1510071 Accepted: April 09, 2019: Published: April 11, 2019

Copyright: (C) 2019 Exarchou EL, et al. This is an open-access article distributed under the terms of the Creative Commons Attribution License, which permits unrestricted use, distribution, and reproduction in any medium, provided the original author and source are credited. 
use, effectiveness, low cost and wide availability, mouthguard use in basketball is not widespread $[2,10]$. The prevailing attitude of basketball players, especially among adolescents, is that they regard the risk of injury, especially dental injury, as low or even non-existent. Moreover, there is a general prejudice against taking any protective measures due to the perception that a mouthguard may either impact negatively on their ability to play or be uncomfortable to wear. These attitudes may explain why previous efforts to promote mouthguards in basketball have largely been ineffective $[7,11]$. Moreover, despite substantial research into the benefits of mouthguard use in contact sports, there has been little focus on basketball, where mouthguard use is limited [2].

Greece and the U.S.A are two countries where basketball is a very popular sport and a large section of the youth population is involved in amateur play. The purpose of the present study is to ascertain the current level of mouthguard use in competitive amateur basketball, comparing Greek and American male basketball players and their general attitudes towards mouthguard protection.

\section{Methods}

Ethical approval for the study was obtained by the Human Research Ethics Committee of the Aristotle University of Thessaloniki, School of Dentistry, Greece, where the study was registered. The convenience sample comprised 215 male amateur basketball players: 107 were from amateur league male teams from two cities of central and northern mainland Greece (Volos, pop. 80,000 and Thessaloniki, pop.1,000,000 respectively); 108 were from male teams participating in the American Midwest Conference of the National Association of Intercollegiate Athletics (NAIA) Division I League (pop. 5,576,606). They were contacted in person and asked to participate in the study during the academic year of 2015/16. Relevant coaches agreed to allow their players to take part in the study during normal training sessions. The average age of participants was $20.5 \pm 4.3$ years (ranging from 16 to 32 ) for Greek players and 20.8 \pm 1.8 years (ranging from 18 to 27 ) for American players. The purpose of the study was fully explained to all participants and informed consent was obtained.

\section{The Questionnaire}

The original questionnaire was prepared in the English language and questions were derived from previous studies published in English which included relevant questions $[7,12,13]$. The original version was translated into Greek by one translator and back into English by another translator to produce a second English version, which was then compared with the original, with minor revisions being made. Subsequently, the second English version was translated into Greek and compared with the first Greek version, with minor modifications.
This was done to check the reliability and validity of the questionnaire in both languages. It was then tested on part of the sample before being distributed to all the athletes. It was designed to be taken autonomously in five minutes or less and each player was asked to fill it in individually to prevent common answers or the sharing of information.

The questionnaire consisted of five demographic questions which required single word answers (age, length of training, team, level and city). All athletes were then asked if they had heard about mouthguards (Yes/No) and to state from where they had first heard about them (teammates, TV, the internet, dentist, coach). Subsequently, athletes were asked: "Do you wear a mouthguard?" (Yes/No). Twelve multiple choice questions followed to ascertain players' attitudes and experience of mouthguard usage, as well as basketball injury experience. For those answering affirmatively (that they use a mouthguard), some of the questions were as follows: "Where did you get your mouthguard (dentist, store, other)?"; "Do you use your mouthguard during training, competition or both?"; "Are you satisfied with its use?"; "Is there something about it that bothers you?"; "If yes, please explain (speech difficulties, breathing difficulties, inconvenience, other)"; "Have you ever suffered a mouth injury while wearing a mouthguard?"; "If yes, do you think it protected you?".

For those who stated that they do not use mouthguards, some of the questions were: "Why have you chosen not to use a mouthguard (cost, lack of comfort, not convinced, negligence, other)?"; "Do you have any plans to use a mouthguard after this survey?"; "Have you ever experienced a dental injury in basketball?"; "If yes, what kind of dental injury (tooth fracture, jaw fracture, tooth laxation, lip laceration, tongue laceration, other)?".

\section{Statistical Analysis}

The data were processed by an SPSS v.15.0 (SPSS Inc., Chicago, IL, USA) database. Descriptive statistics were used. The chi-square test was used to determine the significance of association between variables and Fisher's exact test was used for the determination of the sample. The level of significance was determined to be $p<0.05$.

\section{Results}

According to the results of the questionnaire, $54.8 \%$ of Greek amateur basketball athletes and $86.5 \%$ of USA were informed about mouthguards and the difference between Greek and USA players was statistically significant $(p<0.01)$. Figure 1 presents athletes' sources of information about mouthguards.

Of all 215 players surveyed, $12 \%$ said that they use mouthguards. This breaks down to 13 Greek players (12.1\%) and 13 American players (12\%), which shows 


\section{SOURCE OF INFORMATION}

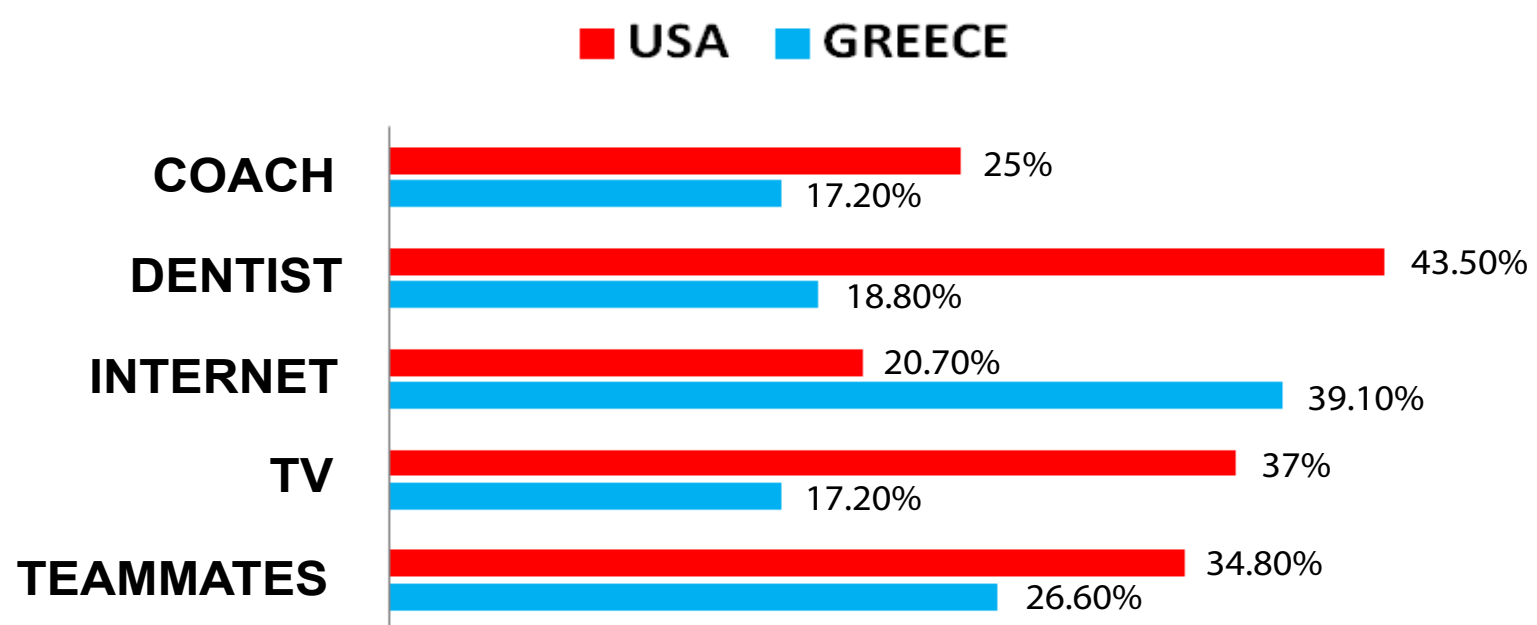

Figure 1: Athletes' sources of information about mouthguards (some totals exceed $100 \%$ due to multiple choices in some cases).

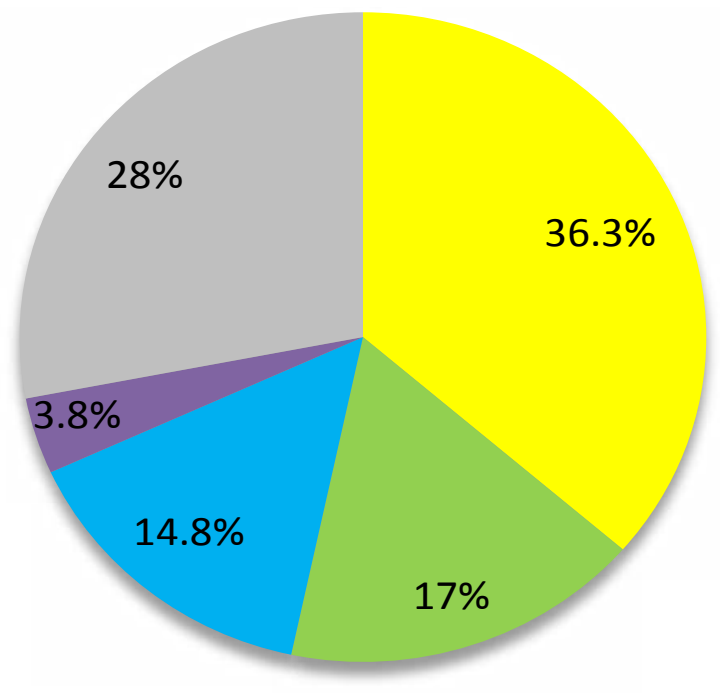

\section{UNCOMFORTABLE $\square$ NO BENEFIT $\square$ NEGLIGENCE $\square$ COST $\square$ OTHER}

Figure 2: Principal reasons for not using a mouthguard from total sample.

no statistically significant difference between Greek and USA players. Those who used mouthguards tended to be older $(p=0.05)$ and to have played basketball for longer $(p=0.05)$. All mouthguard users reported being satisfied with its use. As far as mouthguard complaints by players from both countries are concerned, four felt use was uncomfortable, two players cited difficulties in talking, while one reported breathing difficulties. Of the 13 Greek mouthguard users, ten had experienced injury during mouthguard use, while this was true for three out of the 13 American mouthguard users. In response to the question "Do you think your mouthguard protected you?", affirmative answers were given by nine out of ten Greek and two out of three American players. In Figure 2, the reasons given by players from both countries for not using a mouthguard are shown. In response to the question of interest in future use of a mouthguard, $58.9 \%$ of Greek non-users and $31.1 \%$ of American non-users responded positively $(p<0.01)$ with a statistically significant difference between Greek and USA players, while some players stated that they may be interested in using one in the future, at $5.5 \%$ and $10 \%$ of Greek and American players respectively. $59.1 \%$ of Greek players and $50 \%$ of American players said that they had sustained some kind of orofacial injury while playing basketball. The type of injury suffered by players from both countries is presented in Figure 3.

\section{Discussion}

The overall results of the current study are in 


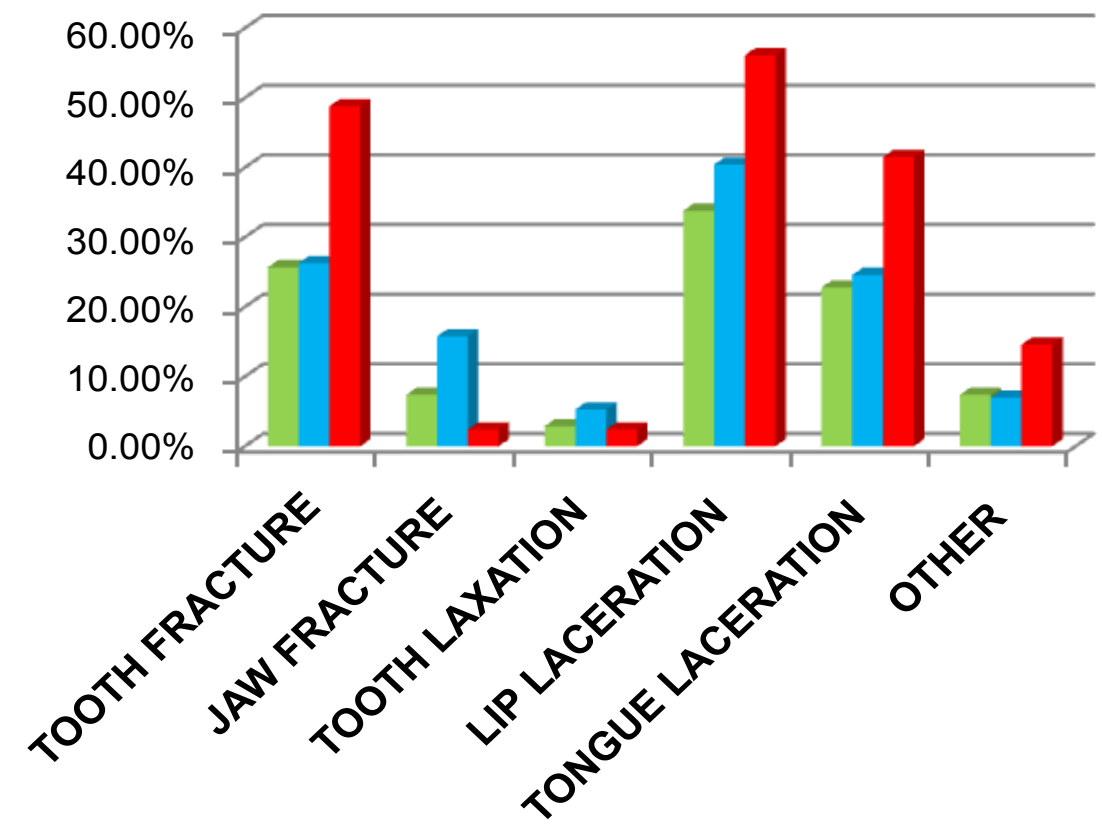

TOTAL SAMPLE

GREECE

USA

Figure 3: Type of injury suffered by players from both countries.

accordance with some previous research indicating the benefits of mouthguard use, as well as the recommendations made by official bodies such as the American Academy of Pediatric Dentistry [6]. The general finding is that the number of mouthguard users in both countries is low. As yet, however, there has been little or no success in using available data to promote policies that will result in higher mouthguard use in basketball [7]. Concerning awareness among basketball athletes, a study in China cited a high proportion of awareness with $80.1 \%$ of players acknowledging the effectiveness of mouthguard use during play [12]. This is close to our findings for the American players' awareness; though awareness among their Greek counterparts was significantly lower (54.8\%).

Comparing how athletes from Greece and the USA are informed about mouthguard use, Greek players were most likely to learn about mouthguards from the internet (39.1\%), while the main source of information for American players was their dentist (43.5\%). On the other hand, Greek dentists have much less involvement in informing players about mouthguards, with only $18.8 \%$ of Greek players citing their dentist as a source of information. A study in China showed even lower percentages, with $6.3 \%$ of basketball players receiving information from their dentists [12].

Many studies confirm that the risk of orofacial and dental injury during sporting activities could be reduced with mouthguard use $[1,10,14,15]$. Furthermore, mouthguard use is recommended for sports including basketball by the American Academy of Pediatric Dentistry [6]. Nevertheless, the present study reveals that only $12 \%$ of Greek and American players alike use mouthguards. Similar studies show either comparable results [16] or even lower mouthguard usage, ranging from $0 \%$ to $6.3 \%[7,10,17]$. More experienced players tend towards mouthguard usage $[7,16]$, which is in agreement with the findings of the present study. Other studies indicate that prior experience of injury is also a factor affecting mouthguard use $[16,17]$.

The current survey supports previous research outcomes regarding common difficulties experienced by mouthguard users. The three principle problems are: lack of comfort $[6,12,17,18]$, breathing difficulties $[6,17]$ and impeded speech $[17,18]$. An additional cause for complaint concerns aesthetic aspects of mouthguards, as outlined in the literature, $[13,18]$, but this was not investigated in the present study. Despite these difficulties, all the players who use a mouthguard responded that they were satisfied with its use.

In the present study, 10 out of 13 Greek mouthguard users had experienced injury compared to 3 out of 13 American players. Of those players from both countries who had suffered an orofacial injury, almost all stated that they thought the mouthguard protected them. This is in line with Tiryaki, et al. who found that $95 \%$ of players felt their mouthguard afforded protection during an orofacial incident [17].

This study identified two main reasons for not wearing a mouthguard: "lack of comfort" and "not convinced". Some studies identify "hadn't thought about it" as a third major cause, which may reflect the insufficient promotion of mouthguards on the part of dentists and professional basketball organizations $[12,16]$. Coaches also seem reticent to promote mouthguard usage, with many never mentioning it to their players $[18,19]$. Additionally, young and amateur players tend not to attach as much importance to mouthguard use as higher-level players do [7]. Cost is another established deterrent to mouthguard usage $[12,18,19]$ with $3.8 \%$ of all basket- 
ball players in the present study citing it as a reason. Compared with factors such as discomfort (36.3\%), this is relatively low.

According to Perunski, et al. some dental injuries require extended, even continuous follow-up treatment, which incurs associated expenses [7]. In the present study, $59.1 \%$ of Greek and $50 \%$ of American non-users reported injury. This was higher than other similar studies $[7,16,20]$. For both Greek and American players, lip laceration was the most common injury, which corresponds with other studies [3]. Fractures reported in other studies ranged up to $17.7 \%$ [17]. The present study identified fractures at $7.4 \%$ and $25.7 \%$ for jaw and tooth fractures respectively. The discrepancy could be explained by the fact that other studies did not specify fracture type. According to literature, the frequency of oral and dental injury occurrence is affected by how much time is spent playing. Wenli Ma found that there was an $80.5 \%$ injury incidence rate among Chinese basketball professionals, while the rate among semi-professionals was a significantly reduced $37.7 \%$, which may be accounted for by the greater time professional players spend on the court [12].

It should be noted that the present study has some limitations. As a convenience sample of teams located in central and northern Greece and the central USA was utilized, the results may not be applicable to amateur teams in other areas. However, the study involved a variety of teams, from both cities and towns, which enhances the external validity of the findings. The study concentrated on male athletes for a purely practical reason-namely, that male basketball teams were more accessible, especially in Greece, perhaps due to the fact that basketball is less popular with females in this country. Additionally, it was considered better to limit the scope of the study to achieve clearer conclusions. Moreover, the questionnaire was completed by all the athletes who were present during the training sessions in which the research was conducted. Another limitation is the low percentage of mouthguard users, at $12 \%$, which was too small a sample to obtain statistically accurate data regarding their follow-up questions.

This notwithstanding, this study adds to the current literature and highlights the need for further research and increased efforts concerning the encouragement of mouthguard use. Some suggested methods for promoting voluntary mouthguard use include sponsorship, endorsement by sporting celebrities and media involvement. However, it is clearly important to educate those working in health care professions, including dentistry, on the need for mouthguard use in sports $[4,21]$. Several studies agree that promoting mouthguard use during training sessions and matches should be a collaborative effort involving dentists, sports physicians, and coaches [7].

\section{Conclusion}

The majority of amateur league basketball players do not use mouthguards, with lack of awareness and perceived inconvenience being the major factors. Dentists, coaches and basketball organizations ought to take a more active role in both the dissemination of information and the promotion of mouthguard usage.

\section{Acknowledgment}

- This study was not funded.

- All the authors declare that they have no conflict of interest.

\section{References}

1. ADA Council on Access, Prevention and Interprofessional Relations, ADA Council on Scientific Affairs (2006) Using mouthguards to reduce the incidence and severity of sportsrelated oral injuries. J Am Dent Assoc 137: 1712-1720.

2. Labella CR, Smith BW, Sigurdsson A (2002) Effect of mouthguards on dental injuries and concussions in college basketball. Med Si Sports Exer 34: 41-44.

3. Stevenson MR, Hamer P, Finch CF, Elliot B, Kresnow M (2000) Sport, age and sex specific incidence of sports injuries in Western Australia. Br J Sports Med 34: 188-194.

4. Schildknecht S, Krastl G, Kühl S, Filippi A (2012) Dental injury and its prevention in Swiss rugby. Dent Traumatol 28: 465-469.

5. Cohenca N, Roges RA, Roges R (2007) The incidence and severity of dental trauma in intercollegiate athletes. J Am Dent Assoc 138: 1121-1126.

6. https://www.aapd.org/research/oral-health-policies-recommendations/prevention-of-sports-related-orofacialinjuries/

7. Perunski S, Lang B, Pohl Y, Filippi A (2005) Level of information concerning dental injuries and their prevention in Swiss basketball: A survey among players and coaches. Dent Traumatol 21: 195-200.

8. Scott J, Burke FJ, Watts DC (1994) A review of dental injuries and the use of mouthguards in contact team sports. Br Dent J 176: 310-314.

9. Kumamoto D, Maeda $Y$ (2005) Are mouthguards necessary for basketball? J Calif Dent Assoc 33: 463-670.

10. Ferrari CH, Ferreria de Mederios JM (2002) Dental trauma and level of information: Mouthguard use in different contact sports. Dental Traumatology 18: 144-147.

11. von Arx T, Flury R, Tschan J, Buergin W, Geiser T (2008) Exercise capacity in athletes with mouthguards. Int $\mathrm{J}$ Sports Med 29: 435-438.

12. Wenli Ma (2008) Basketball players' experience of dental injury and awareness about mouthguard in China. Dental Traumatology 24: 430-434.

13. Lang B, Pohl Y, Filippi A (2002) Knowledge and prevention of dental trauma in team handball in Switzerland and Germany. Dent Traumatol 18: 329-334.

14. Knapik JJ, Marshall SW, Lee RB, Darakjy SS, Jones SB, et al. (2007) Mouthguards in sport activities: History, physical properties and injury prevention effectiveness. Sports Medicine 37: 117-144. 
15. Newsome PRH, Tran DC, Cooke MS (2001) The role of the mouthguard in the prevention of sports-related dental injuries: A review. International Journal of Paediatric Dentistry 11: 396-404.

16. Cornwell H, Messer LB, Speed H (2003) Use of mouthguards by basketball players in Victoria, Australia. Dent Traumato 19: 193-203.

17. Tiryaki M, Saygi G, Ozel Yildiz S, Yildirim Z, Erdemir U, et al. (2017) Prevalence of dental injuries and awareness regarding mouthguards among basketball players and coaches. J Sports Med Phys Fitness 57: 1541-1547.

18. Gardiner DM, Ranalli DN (2000) Attitudinal factors influencing mouthguard utilization. Dent Clin North Am 44: 53-65.
19. Collins CL, McKenzie LB, Roberts KJ, Fields SK, Comstock DR (2015) Mouthguard BITES (Behavior, Impulsivity, Theory Evaluation Study): What drives mouthguard use among high school basketball and baseball/softball athletes 36: 323-334.

20. Levin L, Friedlander LD, Geiger SB (2003) Dental and oral trauma and mouthguard use during sport activities in Israel. Dent Traumatol 19: 237-242.

21. Andrade RA, Evans PL, Almeida AL, da Silva Jde J, Guedes AM, et al. (2010) Prevalence of dental trauma in Pan American games athletes. Dent Traumatol 26: 248253. 